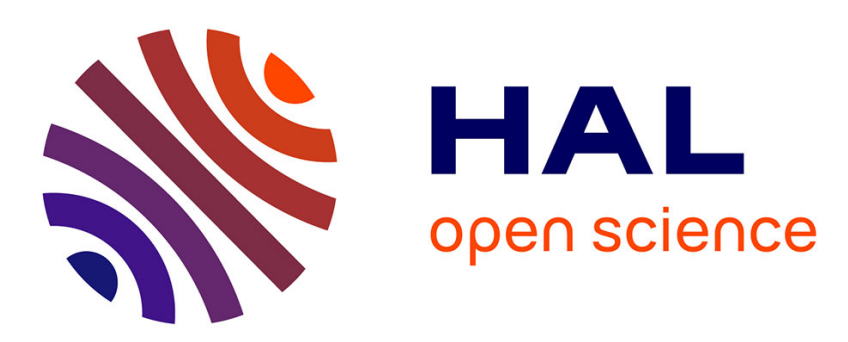

\title{
La construction d'une chaîne associative groupale dans le travail psychanalytique avec les patients psychotiques en groupe: du chaos dissociant à une possible polyphonie Guy Gimenez
}

\section{- To cite this version:}

Guy Gimenez. La construction d'une chaîne associative groupale dans le travail psychanalytique avec les patients psychotiques en groupe: du chaos dissociant à une possible polyphonie. Revue de psychothérapie Psychanalytique de Groupes, 2006, Diversité de psychotéhrapies psychanalytiques de groupe, 54 (1), pp. 71- 91. 10.3917/rppg.054.0217 . hal-01393556

\section{HAL Id: hal-01393556 \\ https://hal-amu.archives-ouvertes.fr/hal-01393556}

Submitted on 7 Nov 2016

HAL is a multi-disciplinary open access archive for the deposit and dissemination of scientific research documents, whether they are published or not. The documents may come from teaching and research institutions in France or abroad, or from public or private research centers.
L'archive ouverte pluridisciplinaire HAL, est destinée au dépôt et à la diffusion de documents scientifiques de niveau recherche, publiés ou non, émanant des établissements d'enseignement et de recherche français ou étrangers, des laboratoires publics ou privés. 


\section{LA CONSTRUCTION \\ D'UNE CHAÎNE ASSOCIATIVE GROUPALE \\ DANS LE TRAVAIL PSYCHANALYTIQUE \\ AVEC LES PATIENTS PSYCHOTIQUES EN GROUPE : \\ DU CHAOS DISSOCIANT \\ À UNE POSSIBLE POLYPHONIE}

GUY GIMENEZ

\section{INTRODUCTION}

Quand on travaille avec des patients psychotiques en situation groupale, cadrée, on peut être étonné que, le plus souvent, chacun d'eux semble moins dissocié qu'en situation individuelle. Non seulement la mise en groupe ne les fait alors ni régresser ni dissocier mais leur permet d'explorer des parts morcelées d'eux-mêmes sans trop de risque. Ceci comme si la mise en commun «protégée » des parts chaotiques de « chaque-un » permettait de construire (ou coconstruire) un espace dans lequel ce qui d'habitude paraît terrifiant, morcelant, abîmant, dissociant, peut enfin être en cet instant et en ce lieu (ici et maintenant) accueilli sans trop de terreur, et gardé sans trop de risque de contamination destructive. Quelquefois même ce qui est accueilli peut être transformé par le groupe (l'ensemble) dans un apprivoisement émouvant. Le clinicien est alors confronté à un aspect spécifique du travail psychanalytique groupal avec les patients psychotiques : une production émotionnelle et verbale se développant chez les membres du groupe, portant la marque du morcellement, de la dissociation de cha- 
cun, et intégrant des productions délirantes et hallucinatoires individuelles. C'est ce que je propose de nommer, avec Evelyn Granjon (2003), les « pointillés associatifs ». Il s'agit de chaînes associatives dissociées des patients, interpénétrées, cacophoniques, conglomérées et non articulées se présentant comme des tentatives autocentrées, narcissiques permettant aux contenus délirants de s'exprimer dans le groupe et d'y trouver, quand cela est possible, refuge (lieu de dépôt) et contenance.

Dans ce texte, je poursuis quatre objectifs.

Je souhaite décrire quelques conditions rendant possible le passage de ce type de production («pointillés associatifs ») à un début de véritable chaîne associative groupale, articulant et nouant les productions verbales individuelles des membres du groupe.

Je voudrais montrer en quoi et dans quelle mesure, les patients psychotiques, en situation de groupe cadrée, peuvent prêter à l'autre des fonctions psychiques non disponibles pour eux-mêmes (comme par exemple la figuration, la scénarisation etc.). Il s'agirait d'une fonction phorique (Kaës, 1993, 1994) particulière (quelqu'un porte quelque chose pour quelqu'un d'autre) ; le groupe devenant un lieu de mise en commun et partage une « coopérative psychique » de fonctions non disponibles à chaque un.

Je souhaite décrire certaines formes d'interventions en groupe (les « interventions double face » (Gimenez, 1996), traduisant le vécu de la séance à la fois au niveau individuel et groupal). Celles-ci permettent de créer des liens à un triple niveau: entre l'individu et le groupe, entre les membres du groupe externe (patients psychotiques), et au niveau intrapsychique (au niveau des groupes internes des patients).

Je voudrais également décrire le travail interne du clinicien rendant possible ces interventions double face. Il s'agit en particulier de repérer ce que j'appelle « les scénarios de base »(Gimenez, 2000) (premier temps), c'est-à-dire les scénarios énoncés dans le groupe et épurés des personnages et compléments qui le qualifient et habillent le scénario. Ces scénarios de base sont structurés comme une phrase : sujet verbe complément sous la forme: «Quelqu'un fait quelque chose à quelqu'un d'autre éventuellement devant un troisième qui regarde. ॥ Il s'agira également d'observer leur succession et leur articulation chez chaque sujet et dans le groupe, dans une séance et entre les séances (deuxième temps).

\section{PRÉSENTATION D’un GROUPE DE PAROLE DE PATIENTS PSYCHOTIQUES}

Je présenterai maintenant une séquence (la vingt-deuxième séance au bout de six mois) d'un groupe de parole constitué de patients schizophrènes. Ce groupe a été mis en place dans une unité de soins de réadaptation (USR) et a fonctionné pendant deux ans et demi. Il s'agit de patients qui, pour la plupart d'entre eux, n'avait pas réussi à sortir 
de l'hôpital de façon durable depuis de longues années et pour lesquels un projet de sortie semblait impensable. Il s'agit d'un groupe hebdomadaire semi-ouvert, constitué, à la période que je vais présenter, par six patients schizophrènes. Les séances durent une heure et demi. La règle de la libre association (on laisse venir ses pensées et on les traduit en mots) dans le respect de chacun, la règle de la discrétion (ce qui est dit dans le groupe reste dans le groupe) sont énoncées de façon explicite au début du groupe. Chacun s'engage également à participer de façon assidue aux séances. Je présentereai une séance qui est très chargée émotionnellement, moment d'un début d'élaboration d'expériences traumatiques importantes pour plusieurs patients.

\section{POSITION PARANOÏDE-SCHIZOÏDE DU GROUPE}

Philippe (27 ans, diagnostiqué psychose infantile) arrive avec un peu de retard. Tout le monde est assis et sa chaise est vide. Les autres semblent l'attendre. Il dit, en s'asseyant, ce que je sens, sur l'instant, ne pas être une métaphore : «J'ai besoin d'une gomme pour ef facer mes parenthèses...»

Isabelle (schizophrène paranoïde de 45 ans ayant fait une décompensation post-partum) assez forte, moulée dans des vêtements très serrés, réajuste depuis quelques instants son gilet trop moulant... Un décolleté plongeant semble laisser Philippe rêveur... Philippe sourit d'un air mi-grivois, mi-gêné, puis reprend sa ritournelle, à mi-voix, comme souvent en début de séance ou dans les moments anxiogènes, devant le groupe qui s'y est habitué : "Je positive et je négative, je positive et je négative, je positive et je négative... »

Je constate que ce mouvement répétitif ne provoque plus d'agacement ni embarras, en moi ou dans le groupe, et je le reçois plutôt comme une berceuse, ou un autobercement rythmique enveloppant... (Avron, 1996). Rachida (schizophrène paranoïde de 40 ans) très sensible aux regards des hommes, a repéré les yeux de Philippe sur le décolleté d'Isabelle (mouvement de rivalité). Comme pour attirer l'attention, elle dit, tendue, en ouvrant grand sa bouche trop maquillée : « J'ai un sosie dans tous les pays. Je suis la plus belle fille du monde, je suis politicienne et actrice! » Elle rit dans une décharge maniaque... et poursuit : « Je vais apocalypser ! » (néologisme). Elle scrute l'assistance qui ne semble pas réagir... J e repère ce double mouvement de mise en avant excitante (la plus belle fille du monde) et sa dilution dans des doubles à l'infini (des sosies dans tous les pays).

Catherine, a, comme souvent, la tête tournée à 90 degrés, ce qui lui donne une allure de personnage pharaonique, mystérieuse... Elle reste silencieuse, comme non concernée par ces échanges. Ses yeux grands ouverts semblent perdus à l'infini, absorbés par un univers précieusement gardé secret... et peut-être impartageable... Isabelle n'a arrêté que 
quelques secondes d'ajuster ses habits qui laboudinent, et, comme un automate, par à-coups, elle reprend son activité... son visage reste très pâle, comme si elle portait un masque...Elle dit, en essayant de rentrer son ventre, en souriant : «Si on mange de trop, on devient pédéraste... D'ailleurs les gens mangent pas beaucoup parce qu'ils font l'amour à l'envers, c'est pour ça que je me tiens mieux à table. » Puis elle poursuit d'un ton grave, plus près de ce qui la préoccupe : « Mon fils a eu un accident de moto... et j'ai dû prendre du Loxapac... le temps qu'il guérisse. Mais ce n'est pas ma faute si on lui a refusé la priorité... » Catherine qui était jusque-là restée silencieuse, renchérit : «Au Brésil j’ai été violée par des Italiens, mon père m’a dit : “Tu n'es plus ma fille.” „) On m'a fait de la sorcellerie, de la Macumba. Après, je savais contrôler le soleil pour que l'explosion ne détruise pas le Brésil.

\section{LA SOUFFRANCE EXPRIMÉE DANS LE GROUPE}

Je repère, sans en mesurer encore assez l'importance, que les scénarios évoqués par les membres du groupe présentent des figurations de plus en plus précises concernant la souf france de chacun (accident de moto du fils d'Isabelle, le viol relaté par Catherine, et son père qui la renie), faisant écho à celle d'autrui, mais de façon encore délirante.

Cette dimension délirante (comme statut de celui qui parle) nous amène ainsi parfois à ne pas saisir le scénario pourtant fort important présenté par le patient. Nicole me regarde. Elle hausse les sourcils ostensiblement, souriant d'un air gêné, comme pour me dire : "ils sont fous... » et s'extraire ainsi du groupe des malades en faisant alliance avec moi sur leur étrangeté à tous... Je la regarde dans les yeux, puis enveloppe l'ensemble du groupe de mon regard, la replaçant ainsi, avec moi, dans l'espace commun du groupe. Je dis : « Nous sommes tous ici et maintenant dans ce groupe, partageant ce que chacun amène, dans le respect de nos différences, dans l'accueil de ce qui peut nous étonner et nous surprendre, des autres et de nous- même... »

Gérald (ancien ingénieur en physique nucléaire de 51 ans) qui a rejoint le groupe de parole depuis 3 séances, dit en regardant ses pieds : «Avant, j’étais ingénieur en physique nucléaire et je croyais qu'il y avait un complot, je crois que je délirais... enfin, je crois... ». Il poursuit : « Mais bon, on me droguait... et j'ai quand même bien vu leur manège, ils ont mis ma fille sur le trottoir... » Je suis touché par la tentative de critique et de prise de distance par rapport à son vécu délirant, dans lequel on le sent se débattre tout en restant encore en partie absorbé (et englué) dedans... J'ai l'impression qu'il demande au groupe d'accueillir sa souf france (mise en dépôt) et le rassurer en lui signifiant qu'il ne délire pas. Je dis (pour Gérald et pour le groupe) «Quelquefois, on peut vivre des choses dif ficiles et douloureuses, 
quelquefois on a du mal à repérer ce qu'est la réalité et ce qu'on a vécu à l'intérieur de soi... le commentaire et le point de vue des autres peut nous aider... à nous repérer , et à mieux nous comprendre, à mieux comprendre ce qu'on a vécu... » Dans le groupe, la phrase de Gérald semble habiter le silence, plein, compact, intense...

\section{NAISSANCE D'UN MOUVEMENT DÉPRESSIF \\ ET SON EXPRESSION CONTRE-TRANSFÉRENTIELLE}

Rachida est visiblement touchée par cette déclaration, centrée sur un aveu dif ficile à exprimer, et renvoyant à sa terreur, déjà évoquée dans ce groupe, « d'être ou de devenir folle pour la vie... » Elle regarde Gérald, comme pour le soutenir avec ses grands yeux écarquillés... Puis, comme pour exprimer un immense découragement, elle place sa tête entre les mains en la tournant répétitivement de droite à gauche en disant : "Je suis fatiguée, exténuée, épuisée... 》 Philippe reprend sans distance par rapport à ce que vient de livrer Rachida (et plus en surface de lui-même) : " Ce qui me fatigue, c'est le dentifrice que je prends... Sensodine et signal +, et Vademecum revitalisant au magnésium... Avant, je prenais Sensodine et ne n'avais pas de problèmes. II y a aussi Extril, celui-là avait un alcaloïde, et je l'ai arrêté parce qu'il me faisait faire des cauchemars... »

Je repère en moi, en écho, une immense fatigue. Cette sensation soudaine d'épuisement semble être le contrecoup d'une grande perte d'énergie à tenter de maintenir ensemble de façon tolérable, coprésents mais non encore articulés, des éléments extrêmement éparpillés, peutêtre même activement déliés... (résultat du rejet, de l'attaque contre les liens). Je me dis qu'une partie de Moi porte (fonction phorique décrite par Kaës), en partie, et dans une certaine mesure, la mise en commun des parts dissociées de chaque un dans le groupe. À travers la figuration de Rachida sur son immense fatigue, je ressens l'écho de ce qui a été mis en dépôt dans l'enveloppe contenante du groupe, fonction à laquelle chaque participant contribue pourtant. Comme dans la phase paranoïde-schizoïde décrite par Melanie Klein (1946), des éléments non encore reliés cohabitent sans encore s'articuler dans ma tête et dans le groupe. Je dis : « Je sens en moi une grande lassitude et cette sensation qu'une multitude de pensées et d'images, demeurent ensemble en moi, sans que je puisse encore vraiment construire une histoire (historisation)... l'histoire de nos échanges et de notre groupe... aujourd'hui. "

\section{ÉVEIL À L'ACCUEIL D'UN PRÉMOUVEMENT DÉPRESSIF (SENTIR LA PERTE)}

Philippe s'occupe depuis un moment de ce que j'imagine être des miettes ou des poussières que l'on peut deviner sur son jean et son pull de laine. Il les saisit une par une et déplace celles de gauche à 
droite et celles de droite à gauche. En même temps, il marmonne de façon à peine perceptible : «Pourquoi j'ose me rappeler du déménagement... L'orphelin pleure, pourquoi ? »Je sens résonner en moi le déménagement, et les pleurs du petitgarçon... Je retrouve des sensations très anciennes, venant d'un autre temps, d'un autre petit garçon, déchiré à chaque nouveau départ... mais je ne dis rien. Je cherche Philippe du regard, comme pour lui tendre une main... Je me laisse toucher par ce mouvement contre-transférentiel, et dont je tente de repérer ce que j'appelle le scénario de base : «Quelqu'un s'en va », « Un petit garçon pleure »... Je me dis que son mouvement répétitif, presque obsessionnel, le protège d'émotions dépressives possiblement trop douloureuses... J e me dis aussi que Philippe porte, peut-être, quelque chose qui le traverse mais appartient aussi au groupe (fonction phorique). J'essaie d'en restituer quelque chose au groupe : Je dis : "Je sens en moi, et dans le groupe, une émotion comme de la peine... comme celle qu'on peut ressentir lors d'une séparation ou d'un moment de profonde solitude. »

\section{PERTE ET ABANDON}

Suit un moment de silence très dense. Puis Gérald reprend la question de 1 a solitude douloureuse. Il dit : « J'ai fait 9 mois en chambre d'isolement (solitude) je cassais tout... j'étais possédé... Mon appartement a été un échec, tout le monde squattait chez moi... Je ne dors pas la nuit, je n'arrive pas à me lever le matin, je voyais des araignées partout, et plein de gens autour de moi... le médecin m'a dit que c'était des hallucinations... » Puis d'un air très contrarié, le visage tendu, il dit : « Mon frère ne m'a pas appelé pour mon anniversaire... » Et il cogne très fort sur le mur avec son poing... se faisant visiblement très mal... Je repère que Gérald a repris, en écho, un scénario relié à un affect dépressif et au vécu de séparation, d'abandon, de solitude ( $\mathrm{CI}$ ) : l'oubli de l'autre. Cet affect est directement lié à l'expérience de crise en CI et de décharge (il cassait tout). L'évocation lui fait revivre cette tension trop forte, insuf fisamment reliée, contenue. La tension est déchargée sous forme d'acte (passage à l'acte, déchar ge par l'acte) dans le cadre du groupe.

L'acting de Gérald est suivi de tension en écho (identification mimétique ?) dans le groupe. Rachida éclate de rire (rire maniaque) ; Isabelle, le visage encore plus figé, dit comme un automate, une phrase énigmatique et délirante : " J'ai bu de l'alcool et j'ai gardé le foi par la bouche, et il m'en reste encore un morceau "; Philippe dit à voix basse, tendu : " Ça sent la merde, les hémorroïdes... "; Isabelle, qui est assise à sa droite, éclate alors aussi de rire, en réaction à la phrase de Philippe, mais comme on réagit à une blague trop grivoise qui 
dérange... ». Catherine effectue des mouvements avec son long cou et sa tête toujours à $90^{\circ}$, son regard est char gé d'une immense tension.

Pendant quelques secondes, je me sens alors vraiment débordé et éparpillé dans ma tentative de mise en sens et mise en mots... Je sens se bloquer ma capacité à penser des idées claires et former des images guidantes, contenantes... pour moi et pour le groupe. Mon superviseur interne (Casement, 1985) non seulement reste muet, mais

semble m'avoir abandonné à jamais... (ultime solitude, désaide) (Winnicott, 1974). Je cherche, comme à tâtons, ce qui pourra faire tenir vivant en moi, mais aussi pour le groupe, le liant nécessaire entre ces nuages de particules de pensées (Bion), dispersées, non encore reliées... et peut-être à jamais éparpillées... C'est le silence en moi... et le brouhaha dans le groupe... comme si le chaos reprenait sa place.

\section{DEVENIR O EN GROUPE : SOI-MÊME TRANSFORMÉ PAR LES AUTRES}

Je sens le multiple en moi (groupe interne), comme si chacune de mes parties ne parlaient pas la même langue (babélisation). Ce que je comprends, après-coup, comme une forme du « devenir O » (Bion, 1970) en groupe (Gimenez, 2003). Alors que je me sens fragile, démuni, et impuissant à aider , aucune interprétation ne se forme dans ma bouche ni dans ma tête. Je sens le groupe glisser dans le vide (signifiant formel) et se perdre sans trouver en moi les ressources pour lui tendre la main ou le tenir, ou le contenir... Je respire doucement, je me reprends et je me connecte aux indices de souf france captés depuis le début de la séance : le fils d'Isabelle qui a eu un accident de voiture, le père de Catherine qui la renie, Gérald qui saisit qu'avant il délirait, Rachida qui est épuisée et découragée, l'orphelin qui pleure et le déménagement, un frère qui oublie d'appeler pour un anniversaire. Puis se forme enfin l'idée pourtant tellement évidente que la tension non contenue par Gérald, réveille, en écho, des tensions en suspens chez chacun. Je regarde chaque participant et, en même temps, le groupe tout entier. Je me sens revenir ici à ma place... Je dis : « Dans ce groupe, on essaie de comprendre en parlant, on essaie de se comprendre en se parlant... en le traduisant par des mots... et des fois quand c'est difficile on peut avoir tendance à exprimer directement par le corps, par des gestes qui sortent trop vite, sans qu'on puisse les contrôler... Et parfois on ne trouve pas les mots, et les autres nous aident à les trouver... Nous pouvons trouver ensemble les mots, les formes, pour traduire ce que l'on vit ici et maintenant dans le groupe. » Le groupe semble se calmer, et Isabelle, et qui est souvent maternanteavec lui, entoure le poing endolori de Gérald de ses deux mains. Celui-ci fait mine de retirer sa main mais l'insistance paisible et ferme d'Isabelle l'amène à abdiquer et lui abandonner cette partie de son corps. Je repère le scénario mis en scène par Isabelle (« quelqu'un materne, enveloppe, accompagne quelqu'un 
d'autre ») ; et le signifiant formel pour Gérald : « une main est enveloppée, câlinée, tenue »).

\section{LE VÉCU DE LA PERTE ET SON RETOURNEMENT PERSÉCUTIF}

Isabelle se remet au bout d'un moment à ajuster ses habits qui la boudinent : comme un automate, par à-coups. Son visage se fige à nouveau, comme un masque... très pâle. Elle dit : «Mon fils a eu un accident de moto... et j'ai dû prendre du Loxapac... le temps qu'il guérisse. Mais ce n'est pas ma faute si on lui a refusé la priorité...» Je suis un instant troublé par la discordance entre le ton de confidence profond d'Isabelle et l'impression qu'elle avait prononcé les mêmes mots, les mêmes phrases, quelques instants avant... Comme si cette répétition niait le temps qui s'était écoulé et que l'on se retrouvait, comme en boucle un long moment en arrière...

Rachida reprend la balle au bond, mais sur un autre registre : « La surveillante à Pontier (nom de son pavillon) m'a volé mes beaux yeux bleus $\gg .$. « Vous avez vu ses yeux maintenant. » Un silence... Des gens entrent en moi et me disent « Caresse-moi » mais moi, je suis la sainte Vierge ». Je me dis intérieurement qu'elle reprend de façon délirante la question de la perte (accident de moto du fils), mais dans un scénario paranoïde... « On vole ou pille une partie du corps », puis en scénario intrusif (une enveloppe est intrusée, pénétrée), et en scénario d'emprise " un être est contrôlé, forcé, dirigé, possédé... » La question de la perte semble ici recentrée sur le lien aux parties de son corps et aux objets partiels : perdre, c'est comme perdre une partie de soi, de son corps (les yeux). Le scénario de base ici, est en parti déscénarisé (mécanisme de descénarisation), sous l'effet de mécanisme d'attaque contre les liens (rejet, envie, destructivité) : on perd quelqu'un, une partie du corps est perdue, on vole une partie du corps. Le mouvement érotisé appréhendé de façon projective ( on me demande d e caresser ») est contre-investi par un mouvement auto-idéalisant ( Je suis la sainteVierge »). Je dis : «On peut être inquiet pour des proches, c'est des fois un peu comme perdre une partie de soi. »

Souhaitant ensuite mettre en mots aussi le mouvement projectif, je poursuis : «Et des fois c'est tellement difficile et douloureux que l'on a l'impression que la douleur ne vient pas du dedans mais du dehors de nous, des autres, et on sent, on ressent, on croit savoir que c'est quelqu'un d'autre qui nous fait souffrir... » Rachida a l'air saisie par mon intervention. Son expression, ses gestes, sont comme en suspens, comme s'il lui fallait du temps pour laisser « reposer » en elle ces mots... et cette expérience... elle semble s'approprier dif féremment ce qui se passe en elle (mouvement introjectif?) 
Philippe reprend : «Je positive et je négative, je positive et je négative... À l'orphelinat j'étais musclé... Juste avant de mourir , ma mère $m$ 'a dit : "Je t'ai fait avec un autre"... » Le groupe est sidéré par ces mots très forts de Philippe. Je suis très ému. Lh silence profond et respectueux suit. Je crois repérer un lien entre les figurations scénarisées de Philippe (déménagement et l'orphelin qui pleure, l'aveux de sa mère que son père n'est pas son père), la fatigue de Rachida, l'accident de moto du fils d'Isabelle, le père de Catherine qui la renie en tant que sa fille... Je repère la thématique perte-décès-dépressiondéception dans le groupe et je dis : «À des moments marquants de notre vie, nos proches sont en danger, s'éloignent de nous, nous quittent, nous déçoivent, nous manquent, et quelquefois nous disent ou font des choses qui nous blessent... »

Philippe devient blanc. Il évoque alors dans le groupe un des deux événements traumatiques qui ont marqué sa famille et sa vie : la défenestration de sa mère quelques mois après celle de sa sœur par la même fenêtre... Il dit : " Ma mère, je crois qu'elle avait l'impression de voir en moi son frère malade quand elle s'est jetée... Elle a ouvert la fenêtre et me regardait. Je regardais la télé, et j' ai dit "saute" au coyote du dessin animé Bipbip... Mais c'est elle qui a sauté. La voisine a sonné.

Mon frère est allé à la porte. Et le voisin a dit qu'elle avait sauté. Je ne suis pas descendu. Je ne l'ai jamais revue. » Je partage et je porte également cette émotion de Philippe et du groupe. À la foisl'affect dépressif de la perte (dans le prolongement des af fects dépressifs pré-sentis par le groupe) et le sentiment d'impuissance extrême (voire de culpabilité) quand un proche se fait du mal, ou essaie de se détruire sous nos yeux. Je repère que Philippe a reconstruit la scène en devenant celui qui a demandé de sauter... dans une confusion entre monde imaginaire (coyote bibip) et la réalité (mère devant la fenêtre). Repensant aux scénarios présentés par Isabelle et Catherine, je m'adresse alors à Philippe et au groupe. Je dis : "Quelquefois des proches souffrent et font des actes très douloureux, ils nous blessent, quelquefois, après, ils ne sont plus, et on se demande, très souvent ce qu'on aurait pu faire pour les aider, leur sauver la vie... ils nous manquent et on s'en veut, on a du mal à comprendre. Philippe laisse couler ses larmes et parvient, pour la première fois, à pleurer en évoquant cet événement déchirant. »

\section{TRAVAIL INTROJECTIF}

Tout le groupe reste en silence, sidéré par la douleur et la peine partagées. Chacun en lien avec ses propres séparations, deuils, peine, en écho... mais gardant cette capacité extraordinaire de mobiliser ce 
qui pourtant en eux est blessé, et d'accompagner l'autre dans une immense douceur et humanité. C'est la fin de la séance.

\section{COMMENTAIRES}

\section{Travail à partir des pointillés associatifs}

Dans un premier temps, les associations sont éparses. La séance se construit comme un tableau impressionniste. Par petites touches de couleurs. Mais je ne vois pas encore d'image. Chacune des lignes associatives, je devrais peut-être dire, chaque pointillé associatif comme nous les appelons avec E. Granjon (2003), se développe, mais sans prendre vraiment en compte celle des autres, sans s'articuler, en pointillé (Gimenez, 2003). Leur discours semble porter la marque de leur morcellement, de leur dissociation.

\section{Liaisons de surface : du morcelé pour du diffracté}

J'observe des articulations en surface, à partir de mots, à partir de sons, de phrases ou de parties de phrases, qui viennent signifier quelque chose dans le délire ou l'univers interne des patients. Alors que j'observe ce qui se passe en moi, je me rends compte que je prends ce qui est éparpillé, morcelé, dissocié pour du dif fracté, c'est-à-dire comme beaucoup plus élaboré que cela n'est (Gimenez, 2003).

\section{Groupalisation comme support à la figuration}

Plusieurs membres du groupe évoquent leurs délires. Ces prises de paroles peuvent être comprises comme des tentatives autocentrées, narcissiques, pour permettre aux contenus délirants de s'exprimer dans le groupe et d'y trouver, quand cela est possible, refuge (lieu de dépôt) et contenance (Gimenez, 2003). Nous observons que tout se passe comme si la coprésence et donc la sommation des lignes associatives de chaque-un (dissociées) n'augmente pas l'ef fet ou le niveau de dissociation (ou de morcellement) pour l'observateur que je suis. contraire, la groupalisation semble permettre l'externalisation et un début de scénarisation, de figuration de ce qui était enclos dans des appareils psychiques individuels incapables de 1 es contenir. Ainsi, la mise en commun, puis l'articulation des pointillés associatifs de chaqueun semble permettre que commence à se construire une chaîne associative du groupe structurée et cohérente dans laquelle chacun trouve un espace pour se dire. 


\section{L'individu comme un groupe}

La méthode que j'ai commencée à élaborer (Gimenez, 1996), et qui consiste à traiter les chaînes associatives des patients schizophrènes comme celles d'un groupe (groupe interne externalisé), m'a amené à considérer la chaîne associative des groupes de patients schizophrènes comme celle d'un ensemble de groupes (ou comme un groupe de groupes), dans lesquels on peut repérer des niveaux de raisonnance (Sandler, 1976) et d'échos multiples (Gimenez, 2003).

\section{De la cacophonie à un début de polyphonie}

Soutenu par mon superviseur interne, j'appréhende la cacophonie associative comme une polyphonie potentielle. Ce qu'elle devient progressivement, en passant par ma psyché, dans l'espace du groupe qui lui fournit un lieu et une enveloppe où loger et contenir ces éléments épars et éparpillants (Gimenez, 2003). Comme préalable délibéré au traitement du matériel de ce type de groupe, j'appréhende et ressens comme diffracté... ce que je sais, d'un autre point de vue, être du morcelé... Cette « illusion ef ficace » rend possible le traitement de la " non-chaîne associative ", du chaos non organisé, issu des "pointillés associatifs » individuels interpénétrés, conglomérés et articulés uniquement en apparence. Les souffrances individuelles, d'abord muettes, sont progressivement réactivées par des associations de chaque-un, et organisées autour de la question du manque, de la perte, de la souf france, de l'abandon, de la trahison, de la mort.Toutes les questions contre lesquelles luttent les défenses psychotiques.

\section{L'attention du clinicien}

J'appréhende toutes les productions des patients comme porteuses potentielles de signification. Ce préinvestissement favorisera leur utilisation comme support d'une possible chaîne associative spécifique (chaîne associative primaire, archaïque). À un moment dans la dynamique de la séance, je me sens découragé et abattu. Je porte contretransférentiellement le mouvement dépressif du groupe qui ne parvient pas encore à se figurer. Puis des scénarios s'articulent autour d'une thématique ou plutôt autour d'un af fect : l'affect dépressif. L'interprétation trouvée-créée est ici celle qui doit revêtir la forme de ce qui était attendue par le patient pour pouvoir le calmer, donner forme et sens à un vécu chaotique, bouleversant, traumatique. Le groupe, en attente, en appel d'un or ganisateur, peut ici trouver -créer une forme apte à contenir (en partie) le vécu subjectif profondément douloureux de chacun des membres (Gimenez, 2003). L'émotion du groupe peut alors 9 
prendre forme, s'articuler et s'or ganiser autour de scénarios évoqués, proposés, quelquefois agis, et très chargés émotionnellement.

\section{Interventions double-face}

Mes interventions ont par moment la fonction d'articulateurs relationnel/émotionnel pour chaque participant et le lien qui les unit. Il s'agit d'organisateur groupalement créé, et porté par le clinicien (fonction phorique, Kaës, 1993), pour le groupe, un or ganisateur intra et inter-psychique, qui rend possible le nouage, l'or ganisation et l'intégration des chaînes associatives individuelles dans une chaîne associative groupale (Kaës, 1994) en train de se constituer.

\section{Émotion commune}

Les participants arrêtent de délirer et se centrent sur l'émotion commune et sur la construction d'un scénario commun. C'est alors que le vécu, la parole, et l'expérience de chaque-un peut être écouté, trouver sa place, et être reçu dans le groupe (Gimenez, 2003). Sont évoquées des situations émotionnelles, intimes et émouvantes de plusieurs, en écho au partage dans le groupe. Ces associations individuelles, intimes, par lesquelles chacun se livre et s'engage émotionnellement, participent à la figuration groupale de ce qui était en suspens en attente et même en appel d'un travail psychique. L 'articulation des chaînes associatives individuelles permettent l'avènement du mouvement dépressif pour un participant et dans le groupe...

\section{POUR CONCLURE}

Traducteur d'af fect de l'un en pensée pour l'autre, le clinicien, investi dans et par le groupe, permet aux interlocuteurs d'explorer, filtrer, réguler et reconnaître sans trop de risque $\mathrm{c}$ e qui était là, souvent en négatif, et mobilisé par la dynamique de la relation. Le clinicien comme être bi-face, devient un lieu de transformation : support de la fonction alpha remobilisée chez le patient et dans le groupe. Le groupe, lieu d'évocation ponctuelle de vécus douloureux et le plus souvent délirants, devient lieu de partage d'une pensée, un lieu d'accueil, $\mathrm{u} \quad \mathrm{n}$ entre-chacun, dans les psychés de chaque sujet qui peuvent enfin s'appareiller et s'accorder. Le travail psychique qui pouvait sembler individuellement trop risqué au dedans, est groupalisé, mis en commun et externalisé sans l'espace du groupe. Ce qui était trop intolérable en chacun est ainsi exploré sans trop de risque dans l'autre, à travers l'autre et les autres (" autre que nous-même ») dans un mouvement de cocréation groupale. 
Avon, O. 1996. La pensée scénique. Groupe et psychodrame, Toulouse, érès. BION, W, R. 1970. L'attention et l'interprétation, Paris, Payot.

Casement, P. 1985. À l'écoute du patient, Paris, Presses Universitaires de France.

GiMENEZ, G. 1996. «La groupalité psychique dans la thérapie individuelle de schizophrènes », dans "Activité de pensée en groupe », Revue française de psychothérapie psychanalytique de groupe, 27, 109-119.

Gimenez, G. 2000. Clinique de l'hallucination psychotique, Paris, Dunod.

GIMENEZ, G., 2003. « Les objets de relation dans la thérapie individuelle et groupale de patients schizophrènes ", Groupes à médiation en pratiques institutionnelles. Revue de psychothérapie psychanalytique de groupe, 41, 41-62.

GRANJON, E. 2003. Communication personnelle.

KAËS, R. 1993. Le groupe et le sujet du groupe, Paris, Dunod.

KAËS, R. 1994. La parole et le lien. Processus associatifs dans les groupes, Paris, Dunod.

KLEIN, M. 1946. " Notes sur quelques mécanismes schizoïdes », dans M. Klein, P. Heimann, S. ; Isaac, J. Rivière, Développements de la psychanalyse, Paris, Presses Universitaires de France, 274-300.

SANDLER, J. 1976. « Contre-transfert et rôle en résonance ", $R F P, \mathrm{n}^{\circ} 3$, maijuin p. 403-412.

WinNICOTT, D.W. 1951. « Objets transitionnels et phénomènes transitionnels », dans Jeu et réalité, Paris, Gallimard, 1975, 7-39.

WinNicott, D.W. 1974. «La crainte de l'effondrement », Nouvelle Revue de psychanalyse, $1975, \mathrm{n}^{\circ} 11,35-44.91$ 